\title{
Editorial
}

Nephrology

Published online: June 19, 2015

\section{Acute Kidney Injury after Transcatheter Aortic Valve Replacement}

\author{
Rajesh Mohandas $^{\text {a,b }}$ R. David Anderson ${ }^{c}$ Carl J. Pepine \\ ${ }^{a}$ Nephrology and Hypertension Section, North Florida/South Georgia Veterans Health System, ${ }^{b}$ Division of \\ Nephrology, Hypertension and Transplantation, and ' $D$ Division of Cardiovascular Medicine, University of Florida, \\ Gainesville, Fla., USA
}

Since its introduction in 2002, more than 150,000 patients have undergone the transcatheter aortic valve replacement (TAVR) globally. The results of randomized trials and observational studies have positioned TAVR as (1) the treatment of choice for inoperable patients with severe symptomatic aortic stenosis and (2) an attractive alternative to surgical aortic valve replacement (SAVR) in high-risk patients [1]. As TAVR results continue to improve with the introduction of smaller delivery systems and other technological advances, it is increasingly considered an option for younger and lower-risk patients.

Acute kidney injury (AKI) frequently complicates SAVR and is associated with increased mortality, infectious complications, and prolonged hospital stay in the short term [2] and serious adverse cardiovascular events and mortality in the long term $[3,4]$. Because cardiopulmonary bypass $(\mathrm{CPB})$, required for a SAVR, is a major predictor of an increased risk for AKI, the less invasive TAVR without CPB was predicted to reduce this AKI risk. However, TAVR requires the use of large delivery devices in the aorta with the potential for associated microembolism, large volumes of radiographic contrast material, and rapid ventricular pacing that induces hypotension, all of which also increase the risk of AKI. Indeed, in the original trial of TAVR leading to FDA approval (PARTNER B trial), AKI occurred only in $4.8 \%$ of cases and AKI requiring dialysis only in $2.9 \%$. However, prac- titioners recognize that AKI frequencies with AVR by either technique are, indeed, high ( $40 \%)$ and vary widely depending on the definition used and the patient characteristics. Clearly, the relative rates of AKI comparing TAVR with SAVR are critically important, particularly as TAVR moves into a younger population with less surgical risk.

Recently, a large analysis ( 12 studies of $>90,000$ SAVR patients and 26 studies of $>6,000$ TAVR patients) confirmed that the frequency of AKI was highly dependent on the definition used [5]. They noted that frequencies of AKI ranged from 3.4 to $43 \%$ with SAVR as $2.5 \%$ required dialysis and from 3.4 to $57 \%$ with TAVR. The independent predictors of AKI were baseline kidney failure, EUROSCORE, diabetes, hypertension, chronic obstructive pulmonary disease, anemia, peripheral vascular disease, heart failure, surgical priority, CPB time, reoperation, use of intraaortic balloon pump, reexploration, contrastvolume, transapical access, transfusion, postoperative thrombocytopenia, postoperative leukocytosis, age, and female sex. The 30-day mortality rates for AKI following SAVR ranged from 5.5 to $46 \%$ (or 3- to 16 -fold higher vs. patients without AKI). Patients developing an AKI after a TAVR had mortality rates ranging from 7.8 to $29 \%$ (or 2 - to 8 -fold higher vs. patients without AKI). Development of an AKI confers up to a 4-fold increase in a 1-year mortality, and the AKI-associated mortality with a SAVR

\section{KARGER 125}

(c) 2015 S. Karger AG, Base

0250-8095/15/0415-0370\$39.50/0
Dr. Carl J. Pepine

Division of Cardiovascular Medicine

University of Florida, 1600 SW Archer Rd.

PO Box 100277, Gainesville, FL 32610 (USA)

www.karger.com/ajn 
appears to be higher vs. TAVR. Finally, the length of hospital stay was longer among patients developing an AKI vs. those without an AKI in both the SAVR and TAVR groups.

In the current issue of this journal [6], a meta-analysis of 3 randomized controlled trials (RCTs) with 1,852 patients and 14 cohort studies with 3,113 patients assessed the AKI risk for TAVR. The pooled data for AKI associated with TAVR suggest $\sim 30 \%$ relative risk reduction compared with SAVR. The sensitivity analysis in RCTs and propensity score-based studies using a standard AKI definition supported an association between TAVR and lower AKI risk (RR: 0.35; 95\% CI 0.25-0.50). Interestingly, associations between TAVR and reduced risks of severe AKI requiring dialysis were not found. The authors concluded that TAVR is associated with a lower AKI risk.

\section{Knowledge Gaps}

Studies examining AKI risk in TAVR have yielded conflicting results due to varying definitions of AKI, procedural differences in TAVR (transfemoral vs. transapical approach), and use of next-generation aortic valves. So, clearly, a uniform definition for AKI after AVR is needed.

While predictive scoring systems have been developed to assess the AKI risk in patients undergoing cardiac surgery, particularly $\mathrm{CPB}$, none have been validated in a large cohort undergoing TAVR. Most studies show that patients who are older or have chronic kidney disease, diabetes, or heart failure are at high risk of AKI. So, should patients with chronic kidney disease who are at high risk of AKI be considered for TAVR? As the long-term durability of transcatheter bioprosthetic valves is not yet known and continues to evolve with the availability of newer valves, SAVR remains the standard, against which newer TAVR technologies will be compared. At least, for the intermediate timeframe, the PARTNER A trial, which randomized high-risk patients to SAVR or TAVR, recently reported that no structural valve deterioration requiring replacement was observed over 5 years and clinical outcomes were comparable [7]. However, before we can state definitively that TAVR leads to less AKI than SAVR, the development of a predictive tool to assess the risk of AKI and a clinical trial randomizing such patients to a SAVR or TAVR is necessary.

Novel biomarkers can help with the early detection of AKI, and while FDA-approved commercial tests are available [8], their role in clinical practice remains to be defined. It would be helpful to better understand the mechanism of AKI in these cases undergoing TAVR, so that more specific management strategies can be developed to prevent or minimize an associated AKI. An ongoing study is assessing the effect of a forced diuresis with a matched hydration in reducing AKI during TAVR (clinicaltrials.gov NCT01866800). Finally, several novel therapies are under study: Erythropoietin + Iron Therapy for Anemic Patients Undergoing Aortic Valve Replacement (EPICURE) (NCT02390102) and Allogeneic Multipotent Stromal Cell Treatment for AKI Following Cardiac Surgery (NCT00733876).

\section{Disclosure Statement}

Dr. Mohandas reports that he had no relevant disclosures. Dr. Anderson is a consultant for Johnson and Johnson and BioSense Webster. Dr. Pepine received funding from the NIH/NCATS Clinical and Translational Science Award to the University of Florida UL1 TR000064.

\section{References}

1 Smith CR, Leon MB, Mack MJ, Miller DC, Moses JW, Svensson LG, et al: Transcatheter versus surgical aortic-valve replacement in high-risk patients. N Engl J Med 2011;364:2187-2198.

2 Chertow GM, Levy EM, Hammermeister KE, Grover F, Daley J: Independent association between acute renal failure and mortality following cardiac surgery. Am J Med 1998;104: 343-348.

3 Hobson CE, Yavas S, Segal MS, Schold JD, Tribble CG, Layon AJ, Bihorac A: Acute kidney injury is associated with increased longterm mortality after cardiothoracic surgery. Circulation 2009;119:2444-2453.

Acute Kidney Injury after Transcatheter Aortic Valve Replacement
4 Généreux P, Kodali SK, Green P, Paradis JM, Daneault B, Rene G, et al: Incidence and effect of acute kidney injury after transcatheter aortic valve replacement using the new valve academic research consortium criteria. Am J Cardiol 2013;111:100-105.

5 Najjar M, Salna M, George I: Acute kidney injury after aortic valve replacement: incidence, risk factors and outcomes. Expert Rev Cardiovasc Ther 2015;13:301-316.

6 Thongprayoon T, Cheungpasitporn W, Srivali N, Ungprasert P, Kittanamongkolchai W, Greason KL, et al: Acute kidney injury after transcatheter aortic valve replacement: a sys- tematic review and meta-analysis. Am J Nephrol 2015, in press.

7 Mack MJ, Leon MB, Smith CR, Miller DC, Moses JW, Tuzcu EM, et al: 5-year outcomes of transcatheter aortic valve replacement or surgical aortic valve replacement for high surgical risk patients with aortic stenosis (PARTNER 1): a randomised controlled trial. Lancet 2015; pii:S0140-6736(15)60308-7.

8 Kashani K, Al-Khafaji A, Ardiles T, Artigas A, Bagshaw SM, Bell M, et al: Discovery and validation of cell cycle arrest biomarkers in human acute kidney injury. Crit Care 2013; 17:R25. 